\title{
Combining complexity measures of EEG data: multiplying \\ measures reveal previously hidden information [version 1;
}

\section{peer review: 2 approved]}

\section{Thomas Burns, Ramesh Rajan}

Department of Physiology, Monash University, Melbourne, 3800, Australia
V1 First published: 01 Jun 2015, 4:137

https://doi.org/10.12688/f1000research.6590.1

Latest published: 01 Jun 2015, 4:137

https://doi.org/10.12688/f1000research.6590.1

\section{Abstract}

Many studies have noted significant differences among human electroencephalograph (EEG) results when participants or patients are exposed to different stimuli, undertaking different tasks, or being affected by conditions such as epilepsy or Alzheimer's disease. Such studies often use only one or two measures of complexity and do not regularly justify their choice of measure beyond the fact that it has been used in previous studies. If more measures were added to such studies, however, more complete information might be found about these reported differences. Such information might be useful in confirming the existence or extent of such differences, or in understanding their physiological bases. In this study we analysed publically-available EEG data using a range of complexity measures to determine how well the measures correlated with one another. The complexity measures did not all significantly correlate, suggesting that different measures were measuring unique features of the EEG signals and thus revealing information which other measures were unable to detect. Therefore, the results from this analysis suggests that combinations of complexity measures reveal unique information which is in addition to the information captured by other measures of complexity in EEG data. For this reason, researchers using individual complexity measures for EEG data should consider using combinations of measures to more completely account for any differences they observe and to ensure the robustness of any relationships identified.

\section{Keywords}

electroencephalograph, complexity, complexity measure, sample entropy, permutation entropy, Lemel-Ziv complexity, fractal dimension, Weiner entropy

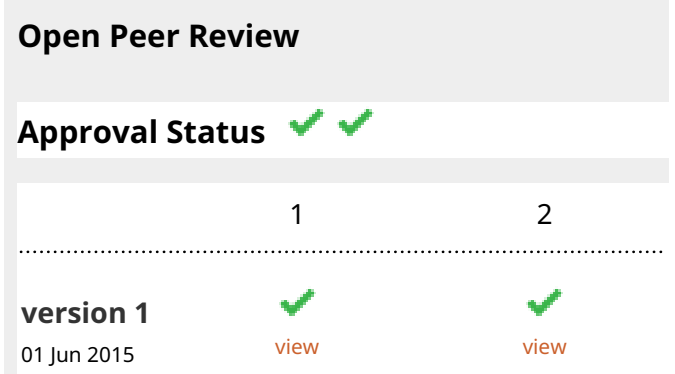

1. Xiao Shifu, Shanghai Jiaotong University School of Medicine, Shanghai, China

2. Alison Pack iD), Columbia University, New York, USA

Any reports and responses or comments on the article can be found at the end of the article. 


\section{incf}

This article is included in the INCF gateway.

Corresponding author: Thomas Burns (t.f.burns@gmail.com)

Competing interests: The authors declared no competing interests.

Grant information: The author(s) declared that no grants were involved in supporting this work.

Copyright: @ 2015 Burns T and Rajan R. This is an open access article distributed under the terms of the Creative Commons Attribution License, which permits unrestricted use, distribution, and reproduction in any medium, provided the original work is properly cited. Data associated with the article are available under the terms of the Creative Commons Zero "No rights reserved" data waiver (CC0 1.0 Public domain dedication).

How to cite this article: Burns T and Rajan R. Combining complexity measures of EEG data: multiplying measures reveal previously hidden information [version 1; peer review: 2 approved] F1000Research 2015, 4:137

https://doi.org/10.12688/f1000research.6590.1

First published: 01 Jun 2015, 4:137 https://doi.org/10.12688/f1000research.6590.1 


\section{Introduction}

Electroencephalography (EEG) is a common, relatively non-invasive research and diagnostic tool. Its one-dimensional signals from localised peripheral regions on the head make it attractive for its simplistic fidelity and has allowed high clinical and basic research throughput. When it comes to interpreting EEG data, investigators have a wide range of analytical tools at their disposal (Dauwels et al., 2010; Delorme \& Makeig, 2004) and in recent years have explored a number of novel relationships between measures of complexity (Cao \& Slobounov, 2011; Dauwels et al., 2011; Jing et al., 2014; Sitt et al., 2014; Susmáková \& Krakovská, 2008; Weiss et al., 2011). Studies which have included complexity measures, however, do not regularly include more than one or two such measures. For example, Dauwels et al. (2011) include the Lempel-Ziv (LZ) complexity measure (Lempel \& Ziv, 1976) - an algorithmicbased measure - and regularity measures, but ignore potential chaotic and fractal measures. This is not to suggest that the LZ complexity measure or that regularity measures are meaningless, nor that chaotic and fractal measures are more or less important than other measures of complexity, but that all may be measuring different features. Thus, for a more complete and robust picture of any relationships found for one complexity measure in EEG data, it might be useful for investigators to include other measures in their analyses.

This study therefore aims to determine whether different measures of complexity of EEG signals correlate, and (if so) to what degree. To do this, a small battery of complexity measures were computed for publicly-available normative data and subsequently analysed for correlations. If some measures were found not to significantly correlate or correlate fully, this would suggest that these measures are detecting unique information which might otherwise have remained hidden to investigators who were computing only a single complexity measure from their data.

\section{Methods}

One thousand, one hundred EEG recordings of 1-second duration from 13 healthy control subjects undergoing an object recognition psychophysics task were obtained from a publicly-available database created by Begleiter (1996) of the Neurodynamics Laboratory, State University of New York Health Center, Brooklyn, United States. The control subjects were selected so as to avoid disease-specific influences. While our sample size was limited by the database, prior studies which used this database reached significance (thus, independent power calculations were not performed). Detailed demographic, subject, recording, and task information can be found in the original study by Zhang et al. (1995). The following complexity measures were calculated in MATLAB for each recording: LZ algorithmic complexity (Lempel \& Ziv, 1976), fractal dimension estimation (FD) (Higuchi, 1988), permutation entropy (PE) (Bandt \& Pompe, 2002), Wiener entropy (WE) (Wiener, 1954), and spectral structure variability (SSV) (Singh, 2011). These measures were chosen on the basis of their broad representation of different conceptions of 'complexity', including informational theoretic, chaotic/fractal, and computational informatic approaches; details of how these measures are calculated and what they measure are well-described by their respective original proposers (Bandt \& Pompe, 2002; Higuchi, 1988; Lempel \& Ziv, 1976; Singh, 2011; Wiener, 1954) and so will not be repeated here (see Data Availability for code details). Many more measures exist than these, however as the principle aim of this paper was to determine if differences exist at all, any differences detected in this small cross-section of measures would sufficiently illustrate this. Results from the complexity measures were analysed by linear regression and significance (considered as $\mathrm{p}<0.05)$ for relationships between pairs of measures was calculated using Pearson product-moment correlation coefficients. For relationships which appeared to have non-linear components when viewing its scatterplot, binomial regression was attempted. Graphs and statistics were generated using MATLAB R2012a (7.14.0.739) and Microsoft Excel 2007.

\section{Results}

Of the ten pairs of measures, eight pairs exhibited highly significant $(\mathrm{p}<0.0001)$ correlations while two pairs - (i) PE and FD, (ii) WE and LZ - did not significantly correlate (Table 1 and Table 2). High degrees of spread were noted among all correlations.

These relationships were visualised using scatter plots (Figure 1 and Figure 2) to help determine if any of these relationships may be non-linear. Two such relationships - (i) LZ and FD, (ii) SSV and FD - appeared to follow a binomial trend (Figure 3), and binomial regression improved these relationships greatly.

\section{Table 1. Pearson (r) correlation matrix for each pair of complexity measures computed for normative EEG recordings.}

\begin{tabular}{|c|c|c|c|c|c|}
\hline & FD & LZ & WE & PE & SSV \\
\hline FD & - & 0.5402 & 0.4155 & -0.0255 & 0.6517 \\
\hline LZ & 0.5402 & - & -0.0472 & -0.1273 & 0.5983 \\
\hline WE & 0.4155 & 0.4155 & - & 0.3469 & 0.5977 \\
\hline PE & -0.0255 & -0.1273 & 0.3469 & - & 0.1672 \\
\hline SSV & 0.6517 & 0.5983 & 0.5977 & 0.1672 & - \\
\hline
\end{tabular}

Table 2. Significance (p) of correlations for each pair of complexity measures computed for normative EEG recordings.

\begin{tabular}{|c|c|c|c|c|c|}
\hline & FD & LZ & WE & PE & SSV \\
\hline FD & - & $<0.0001$ & $<0.0001$ & 0.3990 & $<0.0001$ \\
\hline LZ & $<0.0001$ & - & 0.1174 & $<0.0001$ & $<0.0001$ \\
\hline WE & $<0.0001$ & 0.1174 & - & $<0.0001$ & $<0.0001$ \\
\hline PE & 0.3990 & $<0.0001$ & $<0.0001$ & - & $<0.0001$ \\
\hline SSV & $<0.0001$ & $<0.0001$ & $<0.0001$ & $<0.0001$ & - \\
\hline
\end{tabular}



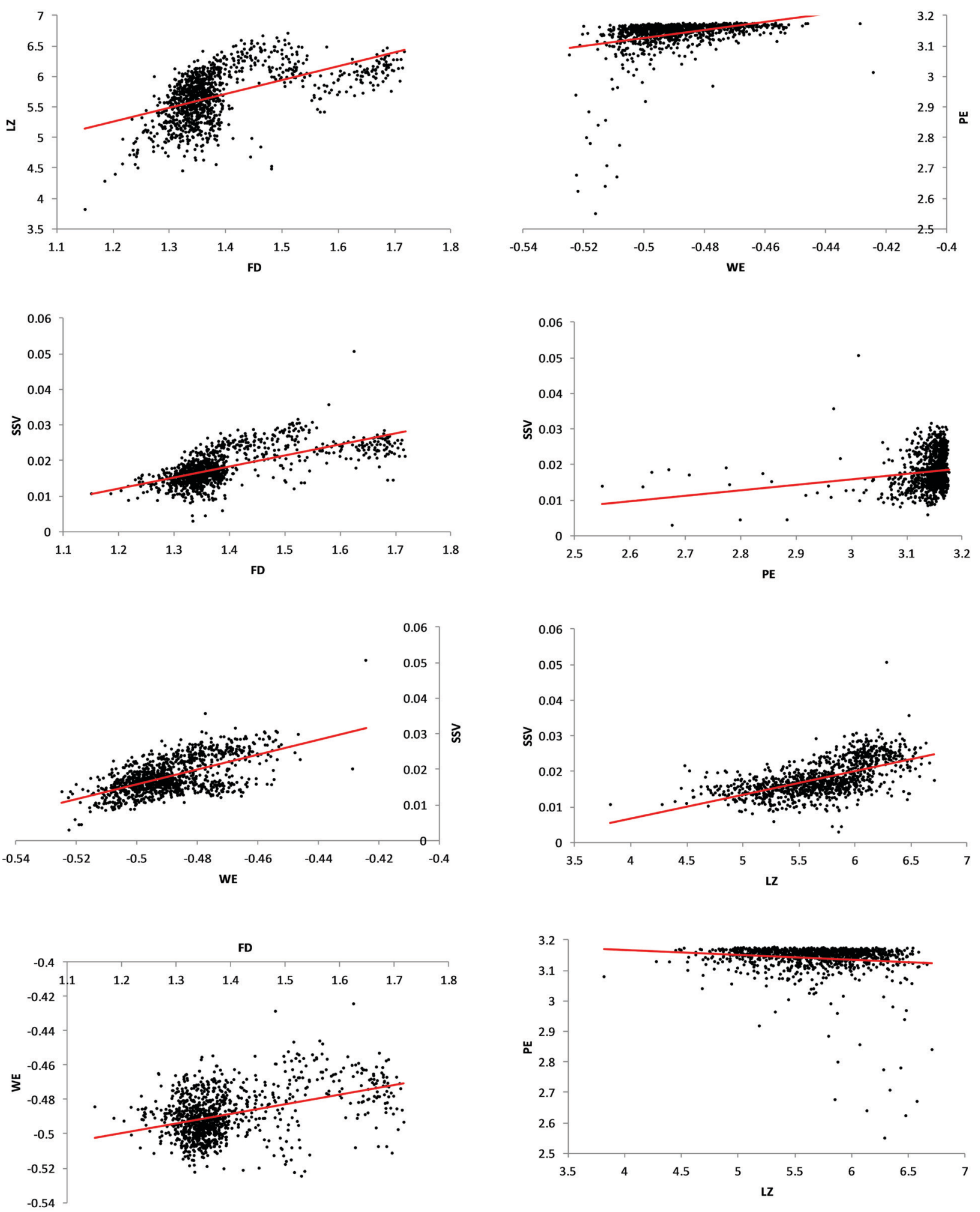

Figure 1. Scatter plots with linear trendlines for pairs of significantly-correlated complexity measures. Eight pairs of complexity measures of the EEG signals had a significant $(p<0.0001)$ correlation. Although the relationships are significant, high degrees of spread are noticeable and some of the relationships may have non-linear components. EEG = electroencephalogram; LZ = Lempel-Ziv algorithmic complexity; $F D=$ fractal dimension estimate (Higuchi method); $P E=$ permutation entropy; SSV = spectral structure variability; WE = Wiener entropy (also known as spectral flatness). 

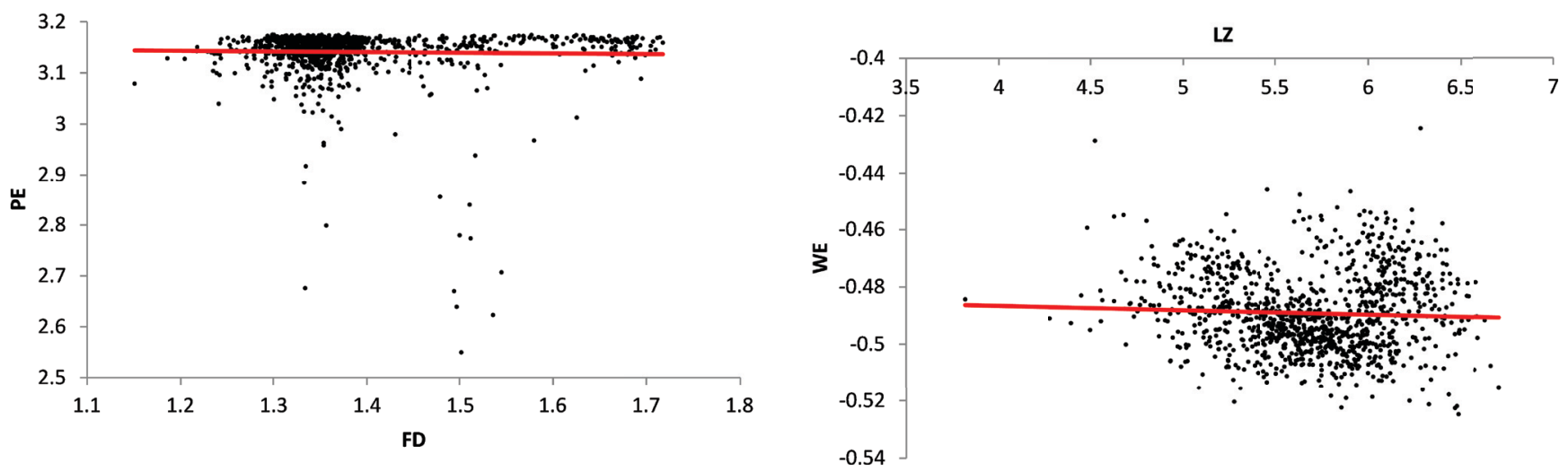

Figure 2. Scatter plots with linear trendlines for two pairs of insignificant, uncorrelated complexity measures. Two pairs of complexity measures of the EEG signals were insignificant and uncorrelated - PE \& FD ( $r=-0.0255, p=0.3990)$ and WE \& $L Z(r=-0.0472, p=0.1174)$. There appears to be no non-linear components or any evidence of a clear relationship between these pairs of measures. EEG =electroencephalogram; $L Z=$ Lempel-Ziv algorithmic complexity; FD = fractal dimension estimate (Higuchi method); $P E=$ permutation entropy; WE = Wiener entropy (also known as spectral flatness).
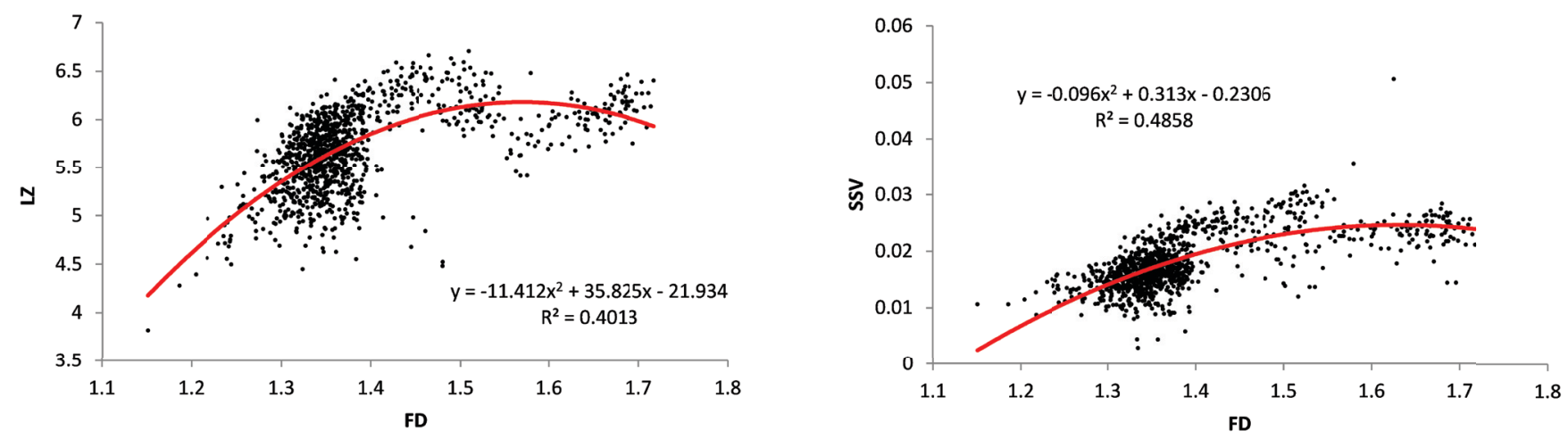

Figure 3. Scatter plots with binomial regression lines for potential non-linearly-related pairs of complexity measures. Two pairs of complexity measures of the EEG signals appeared to have noticeable non-linear relationships: (i) LZ and FD; and (ii) SSV and FD. Although these binomial relationships were - like their linear relationships - significant $(p<0.0001)$, the binomial regressions produced less spread and appear to be truer representations of the relationships. EEG = electroencephalogram; $L Z=$ Lempel-Ziv algorithmic complexity; FD = fractal dimension estimate (Higuchi method); SSV = spectral structure variability.

\section{Dataset 1. Calculated complexity measures for 1100 EEG recordings}

http://dx.doi.org/10.5256/f1000research.6590.d48983

The following data are the results from MATLAB functions which calculated complexity measures for each EEG recording. The following data are the results from MATLAB functions which calculated complexity measures for each EEG recording. ID = identification code as per Begleiter (1996); LZ = Lempel-Ziv algorithmic complexity; FD = fractal dimension estimate (Higuchi method); $P E=$ permutation entropy; WE = Wiener entropy (also known as spectral flatness) (Burns \& Rajan, 2015).

\section{Discussion and conclusions}

Some - but not all - measures of complexity of EEG signals correlate, and to varying degrees of significance, e.g. we found no significant relationship between PE and FD but did find a significant relation between PE and LZ. To the best of our knowledge, this study represents the first report of such complexity measure differences in EEG signals. Of the many complexity measures available to researchers investigating EEG data, overreliance or overconfidence in any single measure therefore seems misplaced. As research groups who have attempted to classify or predict sleep stages or 
conscious states from EEG data have implicitly noted (Susmáková \& Krakovská, 2008; Sitt et al., 2014; Weiss et al., 2011), no individual measure can reliably predict all possibly relevant physiology. Instead, combinations of measures are needed. In the same way, no individual measurement of complexity can reliably predict all possibly relevant complexity.

In part, the results from this short study reflect on a more generalised ambiguity of the concept of 'complexity'. Who is to say, after all, that more is revealed about 'complexity' by FD than LZ? It seems that it cannot be said that either elucidate more or less about 'complexity', since both ultimately treat it in a different way on even a conceptual basis. This further reiterates the primary finding of the present study: by multiplying measures we can reveal information which was previously hidden or unknown to us. However, there are two caveats to this: (1) not all information may be physiologically or otherwise relevant all of the time (or ever); and (2) different datasets may, due to their differences in nature, show different levels of covariance between complexity measures.

It would be interesting for future studies to analyse previouslynoted complexity differences - e.g., between patients with and without Alzheimer's disease (Dauwels et al., 2010) - to determine if these differences were measuring the same difference. Our results suggests they may not be. And if this is the case, more might be gleaned from the available data if more measures were applied in combination. It could even be possible that there exists entirely separate complexity dimensions, along which patients progress at different rates. Such information could therefore contain even more physiological, clinical, or other significance than previously thought.

\section{Data availability}

A copy of MATLAB functions used in this study has been uploaded to GitHub and can be accessed here: https://github.com/tfburns/ MATLAB-functions-for-complexity-measures-of-one-dimensional-signals.

Results from these functions for the EEG data used can be found in the F1000Research repository (see below).

F1000Research: Dataset 1. Calculated complexity measures for 1100 EEG recordings. The data are the results from MATLAB functions which calculated complexity measures for each EEG recording, 10.5256/f1000research.6590.d48983 (Burns \& Rajan, 2015).

\section{Author contributions}

TB conceived, wrote, and performed all analyses pertaining to the manuscript. RR assisted in the development of the conceptual and methodological components of the analyses. All authors have read and agreed to the final content of the manuscript.

\section{Competing interests}

The authors declared no competing interests.

\section{Grant information}

The author(s) declared that no grants were involved in supporting this work.

\section{Acknowledgements}

Thank you to MATLAB community members for their assistance with some of the functions used for calculating complexity measures in this paper.
Bandt C, Pompe B: Permutation entropy: a natural complexity measure for time series. Phys Rev Lett. 2002; 88(17): 174102.

PubMed Abstract | Publisher Full Text

Begleiter H: EEG data set of healthy and alcoholic adults completing

psychophysics tasks [data-set]. Machine Learning Repository, University of California, Irvine [distributor]. 1996.

Reference Source

Burns T, Rajan R: Dataset 1: Combining complexity measures of EEG data: multiplying measures reveal previously hidden information. F1000Research. 2015.

Data Source

Cao C, Slobounov S: Application of a novel measure of EEG non-stationarity as 'Shannon- entropy of the peak frequency shifting' for detecting residual abnormalities in concussed individuals. Clin Neurophysiol. 2011; 122(7): 1314-21. PubMed Abstract | Publisher Full Text | Free Full Text

Dauwels J, Srinivasan K, Ramasubba Reddy M, et al.: Slowing and Loss of Complexity in Alzheimer's EEG: Two Sides of the Same Coin? Int J Alzheimers Dis. 2011; 2011: 539621.

PubMed Abstract | Publisher Full Text | Free Full Text

Delorme A, Makeig S: EEGLAB: an open source toolbox for analysis of singletrial EEG dynamics including independent component analysis. $J$ Neurosci Methods. 2004; 134(1): 9-21.

PubMed Abstract | Publisher Full Text

Dauwels J, Vialatte F, Cichocki A: Diagnosis of Alzheimer's disease from EEG Signals: where are we standing? Curr Alzheimer Res. 2010; 7(6): 487-505. PubMed Abstract | Publisher Full Text

Higuchi T: Approach to an irregular time series on the basis of the fractal theory. Physica D: Nonlinear Phenomena. 1988; 31(2): 277-83. Publisher Full Text

Jing $L$, Jiaqing $Y$, Xianzeng $L$, et al:: Using Permutation Entropy to Measure the Changes in EEG Signals During Absence Seizures. Entropy. 2014; 16(6): 3049-61. Publisher Full Text

Lempel A, Ziv J: On the complexity of finite sequences. IEEE Transactions on Information Theory. 1976; 22(1): 75-81.

Reference Source

Singh NC: Measuring the 'complexity' of sound. Pramana. 2011; 77(5): 811-6. Publisher Full Text

Sitt JD, King JR, El Karoui I, et al:: Large scale screening of neural signatures of consciousness in patients in a vegetative or minimally conscious state. Brain. 2014; 137(pt 8): 2258-70.

PubMed Abstract | Publisher Full Text

Susmáková K, Krakovská A: Discrimination ability of individual measures used in sleep stages classification. Artif Intell Med. 2008; 44(3): 261-77.

PubMed Abstract | Publisher Full Text

Weiss B, Clemens Z, Bódizs R, et al:: Comparison of fractal and power spectral EEG features: effects of topography and sleep stages. Brain Res Bull. 2011; 84(6): 359-75

PubMed Abstract | Publisher Full Text

Wiener N: In The human use of human beings: Cybernetics and society. Boston Houghton Mifflin. 1954; 15-27.

Reference Source

Zhang $\mathrm{XL}$, Begleiter $\mathrm{H}$, Porjesz $\mathrm{B}$, et al:: Event related potentials during object recognition tasks. Brain Res Bull. 1995; 38(6): 531-8.

PubMed Abstract | Publisher Full Text 


\title{
Open Peer Review
}

\section{Current Peer Review Status:}

\section{Version 1}

Reviewer Report 24 September 2015

https://doi.org/10.5256/f1000research.7077.r8846

(C) 2015 Pack A. This is an open access peer review report distributed under the terms of the Creative Commons Attribution License, which permits unrestricted use, distribution, and reproduction in any medium, provided the original work is properly cited.

\begin{abstract}
Alison Pack
Neurological Institute, Columbia University, New York, NY, 10032, USA

This is a well written report addressing the utility of multiple measures used to evaluate EEG. EEG continues to be a valuable tool for research and clinical work. This paper nicely assesses through statistical analysis whether unique features of EEG can be helpful. The methods and analysis are well formulated. The conclusions are clearly articulated and are supported by research findings.

Competing Interests: No competing interests were disclosed.

I confirm that I have read this submission and believe that I have an appropriate level of expertise to confirm that it is of an acceptable scientific standard.
\end{abstract}

Reviewer Report 25 June 2015

https://doi.org/10.5256/f1000research.7077.r8965

(C) 2015 Shifu X. This is an open access peer review report distributed under the terms of the Creative Commons Attribution License, which permits unrestricted use, distribution, and reproduction in any medium, provided the original work is properly cited.

\section{Xiao Shifu}

Alzheimer's Diagnosis and Treatment Centre, Shanghai Jiaotong University School of Medicine, Shanghai, 200030, China

This is an interesting article. The authors computed five complexity measures which were broadly representative, aiming to determine the correlations and differences between measures of complexity used in EEG signals study. Just as the findings of this study, more information can be revealed by multiplying measures instead of single complexity measures. Furthermore, the methods of combinations of complexity measures can be applied in future studies such as 
Alzheimer's disease. With the coming of big data era, we need to adopt effective means to mine information hidden in many diseases. Perhaps, this paper gives us some reference.

Competing Interests: No competing interests were disclosed.

I confirm that I have read this submission and believe that I have an appropriate level of expertise to confirm that it is of an acceptable scientific standard.

The benefits of publishing with F1000Research:

- Your article is published within days, with no editorial bias

- You can publish traditional articles, null/negative results, case reports, data notes and more

- The peer review process is transparent and collaborative

- Your article is indexed in PubMed after passing peer review

- Dedicated customer support at every stage

For pre-submission enquiries, contact research@f1000.com 